\title{
LÓGICA, CIENCIA Y FILOSOFÍA EN VICENTE MUÑOZ DELGADO (1922-1995)
}

\author{
José Luis Fuertes Herreros \\ Universidad de Salamanca
}

\section{RESUMEN}

Se pretende ofrecer una aproximación a la obra de Vicente Muñoz Delgado. Se muestran las distintas etapas en la génesis de su obra y pensamiento, así como también, los núcleos y las claves sobre las cuales se estructura.

Palabras clave: Muñoz, Lógica, Ciencia

\begin{abstract}
Our aim is to offer an approach to the work of Vicente Muñoz Delgado. The different stages of the production of his work and thought, as well as the nuclei and keystones on which it is structured.
\end{abstract}

Key words: Muñoz, Logic, Science

\section{INTRODUCCIÓN}

Sobre Vicente Muñoz Delgado tenemos hasta el presente una muy buena recopilación de su fecunda y extensa obra, unos 240 trabajos entre artículos y libros publicados, y unas 690 recensiones. Ésta nos ha llegado en cuatro momentos distintos, pero ha sido elaborada con la misma metodología, esto es, se han recopilado acumulativamente sus publicaciones según un orden cronológico.

La primera se la debemos a Daniel Hernández Puertas, que en el curso 1972-73 presentó en la Facultad de Filosofía de la Universidad Pontificia de Salamanca una memoria de licenciatura sobre la vida y la obra intelectual del profesor Vicente Muñoz Delgado, que llegaba hasta mediados del año 73.

La segunda fue la propia Reseña bio-bibliográgica del prof. Vicente Muñoz Delgado, publicada en Salamanca, Imprenta Calatrava, 1977, por el mismo Vicente Muñoz sobre los datos de la anterior tesina, como indica en la contraportada: «Publicamos ahora un extracto de dicha tesina, completando algunos datos hasta junio de 1977».

La tercera fue elaborada por la redacción de la revista Cuadernos Salamantinos de Filosofía, bajo el título «Profesor Vicente Muñoz Delgado. Biobliografía». En ella, además de utilizar los 
materiales anteriores, se sirvió de «un completo dossier personal, que le fue entregado amablemente por el propio Dr. Vicente Muñoz Delgado», asegurando al lector «una Bibliografía completa (al menos, en el aspecto científico) de la copiosa producción del homenajeado, hasta octubre de 1979 en que se escribe esta nota». ${ }^{1}$ Era el año 1979 y Cuadernos Salmantinos de Filosofía ofrecía el número VI, correspondiente a dicho año, como un número-homenaje para conmemorar los 25 años que Vicente Muñoz desempeñaba la cátedra de Lógica, 1954-79, de la Universidad Pontificia de Salamanca.

La cuarta se debe a la revista Estudios, que en 1995 prepara un número Homenaje a Vicente Muñoz Delgado. Estando a punto de aparecer dicho número se produjo la muerte repentina de éste, el 20 de diciembre, viéndose obligada a indicarlo en una nota. En este volumen aparecía la última recopilación bajo el título Resumen de lá labor cultural del Prof. Vicente Muñoz Delgado, Catedrático de la UPS, centrándose, principalmente, en continuar la tarea que Cuadernos Salmantinos había dejado en 1979, y llevándola hasta ese año $95 .^{2}$

Es probable que todavía queden varios trabajos sin recoger, así como algunos inéditos, de los cuales hay constancia que están sin publicar. Con el presente artículo no pretendo completar lo que, en líneas generales y con muchísimo celo, ya ha sido reseñado. Intento, más bien, ofrecer una aproximación a la obra de Vicente Muñoz Delgado, destacando las distintas etapas en la génesis de su obra y de su pensamiento, así como los núcleos y las claves sobre las cuales se estructura, proyectando todos estos aspectos en lo acontecido en estos últimos 50 años, tanto en el campo de la lógica y de la ciencia como en el de la filosofía.

Con ello, es posible que asistamos, sorprendidos, a través de la obra de Vicente Muñoz, ala necesaria renovación de la lógica, de la ciencia y de la filosofía, que se va a ir produciendo a partir de los años cincuenta, y viceversa, veremos cómo él, con sus escritos, va contribuyendo a renovar estos campos del saber. Quizá su obra nos pueda ayudar en esa tarea. No sólo se puede descubrir este tránsito a través de él, sino que, a su vez, el mismo aparecerá como uno de los impulsores de aquel camino de renovación que, solidariamente con muchos otros, acabará transformando el hacer lógico, filosófico y científico de la España de la postguerra, para llegar al florecimiento que a partir de la década de los años 70 comenzó a vivirse.

\section{UNA SITUACIÓN COMO PUNTO DE PARTIDA}

Vicente Muñoz Delgado, que había nacido en Marzás-Puenteambía (Baños de Molgas, Orense) en 1992, ingresa a los 11 años en la Orden de la Merced, estudiando Humanidades en el colegio seminario que estos padres mercedarios tenían en Sarria (Lugo), y luego Filosofía y Teología en el monasterio de Poyo (Pontevedra). En 1945 le vemos ejerciendo de profesor auxiliar de Filosofía, Lengua y Literatura griega en el propio monasterio de Poyo.

En 1947 obtiene la licenciatura en Teología por la Universidad Pontificia de Salamanca, y en 1947-48 aparece de nuevo como profesor, ahora de Teología, en el monasterio de Poyo. En 194850 se licencia y doctora en Filosofía por la Universidad de Santo Tomás de Roma (Angelicum).

1 «Profesor Vicente Muñoz Delgado. Biobliografía», Cuadernos Salmántinos de Filosofía, VI (1979) p. 30 , «Nota».

2 Homenaje a Vicente Muñoz Delgado, Estudios (1995), pp. 197-230. 
Durante 1950-54 es profesor suplente de Lógica y Ordenación de las Ciencias en la Universidad Pontificia de Salamanca y en 1954 es nombrado profesor ordinario, catedrático de Lógica y Ordenación de las Ciencias, de la Facultad de Filosofía de dicha Universidad.

Publica en estos años de 1947-54 los siguientes trabajos: ${ }^{3}$

1947 «Los Comentarios a la primera parte de Santo Tomás de Fray Jerónimo Pérez († 1549)».

1948 «Averroísmo de Averroes». «La Asunción de María en el P. Oña, clásico español». «Hambre insaciable. Un mito de Platón». «Humanismo mercedario». «Religión y devoción». «Natural y sobrenatural». «La Eucaristía, alimento del alma».

1949 «El trabajo es obligatorio para todos». «Importancia de las excavaciones en las grutas de la Basílica del Vaticano». «Una gloriosa fecha: bodas de diamante de la ordenación sacerdotal y primera misa del P. A. Londei, mercedario». «Las esperanzas y frutos del Año Santo».

1950 «La indulgencia jubilar del Año Santo 1950». «Los esplendores del Año Santo». «Nápoles Mercedaria. La Archicofradía de la Merced de Nápoles». "Gregorio de Arsicio, reformador de la facultad de Artes en Salamanca», y el libro El influjo del entendimiento sobre la voluntad según Francisco Zumel (†1607).

1951 «Las invitaciones de Dios al orden sobrenatural».

1952 «Revalorización de la persona». «La Eucaristía y la Paz». «La "Mística Ciudad de Dios" y los mercedarios». «La lógica predicativa y la lógica de la inherencia». «El existencialismo y la encíclica Humani Generis».

1953 «Ortega y Gasset en España», y el libro Zumel y el molinismo.

1954 «La enseñanza de la lógica en Salamanca durante el siglo XVI». «Nuevos documentos acerca de las controversias "de auxiliis". Problemas y sugerencias». «Francisco Zumel y La Inmaculada».

Los titulos muestran, en buena medida, las inquietudes que estaban presentes en Muñoz Delgado y, a la par, reflejan el ambiente intelectual que se respiraba tanto en sus aulas universitarias como de modo general en España, tensionado, fundamentalmente, entre lo religioso y la neoescolástica. Responde esta situación a la propia descripción que el mismo Muñoz Delgado hará unos cuantos años más tarde:

En 1940, se funda la Universidad Pontificia de Salamanca y, en 1946, se crea la Facultad de Filosofía que, hasta 1963, es puramente eclesiástica. El primer profesor de lógica es Pedro Urbano Gil de Ortega, procedente del Seminario de Vitoria, que solamente enseña durante el curso 194647. Tanto en Vitoria como en Salamanca utiliza los manuales de Paul Geny, su profesor en la Gregoriana, y A. Naber, recomendando a J. Fröbes con moderadas alusiones a la lógica moderna a través de Bochenski. Le sucede Nolasco Vázquez Pájaro (1948-49), formado en la Universidad Central de Madrid, utilizando las obras de Juan David García Bacca.

Pero el texto base de sus cursos y programas lo constituye el manual de F.X. Maquart y, en mucho menor grado, el de Pfänder y Romero-Pucciarelli, de corte fenomenológico. En el curso 1950-51 le sucede Vicente Muñoz Delgado, que continúa hasta el presente. ${ }^{4}$

3 Para las referencias completas y la localización de los distintos trabajos de Vicente Muñoz remito, hasta el año 1978 inclusive, a Cuadernos Salmantinos de Filosofia, VI (1979), pp. 7-30, y de 1979 a 1995, al Homenaje a Vicente Muñoz Delgado de la revista Estudios (1995), pp. 197-230.

4 Vicente Muñoz Delgado, «Para la historia de la lógica en España (1939-1969)», en Actas del II Seminario de Historia de la Filosofía Española, p. 218. En adelante, citaré este trabajo como Actas II. 
Este mismo ambiente, con algunos matices, es el que había en las tres Universidades del Estado en las que se enseñaba Filosofía: Madrid, Murcia y Barcelona.

En Madrid «D. Leopoldo-Eulogio Palacios es el catedrático de lógica de la Universidad [...] durante estos años. En 1942 es catedrático de Instituto y encargado del Curso de lógica, cuya cátedra gana en 1944, y la desempeña hasta el presente. Da una enseñanza tradicional, basada en Gredt y Juan de Santo Tomás, con atención a los grandes lógicos ibéricos del pasado». ${ }^{5}$

«Por Murcia pasa Huidobro de la Iglesia, muy interesado en lógica. En 1953 está encargado del curso de la cátedra de lógica Enrique Antón Cano, con 3 alumnos. No parece haber nada interesante en orden a la lógica, aunque en filosofía hay las figuras de Muñoz Alonso, A. González y Tierno Galván».6

Y lo mismo sucedía en Barcelona, «a pesar de la tradición de J.D. García Bacca, no parece sobresalir mucho durante este periodo. Fermín de Urmeneta está encargado de la cátedra de lógica entre $1947-59 » ?$ ?

Sin embargo, de esta situación en la que se encontraba inmerso y que de modo cercano le tocaba vivir, trataría de salir, pues «no se podía seguir así». Quizá por emulación con la propia Universidad de Salamanca, en donde Gustavo Bueno daba cursillos de lógica moderna, a los que asiste, al igual que a algunos de Miguel Cruz Hernández, ${ }^{8}$ y quizá, o ambas cosas a la vez, por las novedades que comenzaban a llegar y a conocerse a través de la revista Theoria, que había fundado Miguel Sánchez Mazas y cuyo primer número se publicaba en abril de 1952, ${ }^{9}$ el caso es que Muñoz decidió marchar a estudiar fuera.

\section{NUEVAS PERSPECTIVAS, 1954-60}

Durante 1954-56 cursa estudios intensivos de Lógica matemática en la Universidad de Lovaina bajo la dirección de Roberto Feys, ${ }^{10}$ José Dopp, Alfonso Borghers y Juan Ladrière, simultaneando las clases en la Facultad de Filosofía y en el de la Ciencia (sección Matemáticas). Participa en el Centre pour la Recherche de logique de Lovaina y muy frecuentemente en el de Bruselas.

Después, tras integrarse a su cátedra en la Pontificia de Salamanca durante los cursos $56-57$ y 57-58, se trasladaría hasta finales del verano de 1960 a Estados Unidos, donde sigue cursos intensivos de Logica matemática, Filosofía del lenguaje y Filosofía de la ciencia en la Western Reserve University de Cleveland (Ohio, EE.UU.) bajo la dirección de los profesores Isaac Levi y J.M, McLellan, y en la Georgetown (Washington-EE.UU.) sobre Logica matemática, Lógica antiguomedieval y comparación entre ambas.

5 Actas $I I$, p. 220.

6 Ibidem.

7 Ibidem, p. 221.

8 Vicente Muñoz Delgado, Reseña biobliográfica del Prof. Vicente Muñoz Delgado, Salamanca, Imprenta Calatrava, 1977, p. 4.

9 V. Muñoz se hará eco de los artículos que en Theoria publica M. Sánchez Mazas y J. Rey Pastor. Cf. Actas II, pp. $256,314-315$ y 271.

10 Sobre Feys, Actas II, pp. 237-238. 
Tras esto, se reincorpora definitivamente a su cátedra de Lógica; en octubre de 1960. Como dice Muñoz Delgado: «la auténtica renovación de la enseñanza de la lógica (en la Universidad Pontificia de Salamanca) se comienza hacia 1957, a la vuelta de Lovaina del titular, y, sobre todo, en 1960, al regreso de la norteamericana Western Reserve University». ${ }^{11}$

Este giro y renovación, que progresivamente se ha ido dando en sintonía con las corrientes europeas y norteamericanas, comienza a hacerse presente en el nuevo modo de abordar y presentar la lógica. Las publicaciones que efectúa ya en estos años, así nos lo manifiestan. En este sentido tenemos:

1956 «El algebra de la lógica». «Lógica escolástica y lógica moderna».

1957 «Un nuevo modo de presentar la formalización de la ciencia». «La interpretación formalista de la silogística de Aristóteles». «La obra lógica de Ernesto Schröder». «Sentido de la obra lógica de Ernesto Schröder. El problema de la lógica de clases». «La lógica antigua y medieval a la luz de la logística».

\section{IMPULSAR LA NECESARIA RENOVACIÓN, 1960-70}

Durante esta década de 1960-70, y según el propio sentir de Vicente Muñoz Delgado, se va producir la «renovación de la lógica».12

Dicha renovación es la que se va extendiendo por todo el ámbito universitario, tanto en las nuevas orientaciones que se van dando a las cátedras de Lógica de las Universidades de Madrid, Barcelona y Valencia, como en los estudios que se van suscitando. La apertura y los nuevos aires intelectuales pasan, ahora, por un acercamiento a la lógica.

En Madrid, ahora, publicará Leopoldo-Eulogio Palacios, su Filosofía del saber, $1962^{13}$ y su traducción y prólogo crítico a la obra de Menne Alonso, Introducción a la lógica, $1969 .{ }^{14} \mathrm{En}$ Valencia Manuel Garrido dará un impulso decisivo a la lógica con su toma de posesión en 1962, que culminará con la fundación de la revista Teorema, 1971, y con la traducción en 1970 por Carmen García Trevijano del manual de Benson Mates, Lógica matemática elemental. ${ }^{15}$ En Barcelona, entre 1959 y 1965, Jorge Pérez Ballestar se empeñará en esta renovación, siguiéndole Jesús Mosterín, adjunto a partir de 1960 y catedrático en 1972, que culminará esta tarea y que en 1970 publicará su Lógica de primer orden ${ }^{16}$ También en Barcelona, en la Facultad de Ciencias Sociales y Económicas está, ahora, Manuel Sacristán, que publica Introducción a la lógica y análisis formal. ${ }^{17}$

11 Ibídem, p. 218.

12 Ibídem, p. 219. Para una perspectiva más amplia de lo que voy a señalar véase, M. Garrido, «La lógica matemática en España (1960-70)», Teorema, 2, n. ${ }^{\circ} 6$ (1972), pp. 119-132.

13 Madrid, Gredos, 1962.

14 Madrid, Gredos, 1969, 1974, 1976...

15 Madrid, Tecnos, 1974. Segunda reimpresión revisada, 1977, con una completa bibliografía.

16 Barcelona, Ariel, 1970. Íd., Teoría axiomática de conjuntos, Barcelona, Ariel, 1971.

17 Barcelona, Ariel, 1964, 1969, 1970, 1973. 
Hay que señalar, también, y entre otros, los trabajos que publican en estos años los profesores Javier Muguerza, ${ }^{18}$ Emilio Lledó,${ }^{19}$ Maximiliano Fartos,${ }^{20}$ Alfredo Deaño ${ }^{21}$ y Teodoro de Andrés. ${ }^{22}$

Los centros universitarios de la Iglesia comienzan, también, por estos años 60 su necesaria renovación. Comillas, Navarra, en donde Jorge Pérez Ballestar traducirá y adaptará el manual de E. Agazzi, Lógica simbólica, ${ }^{23}$ además de la Universidad Pontificia de Salamanca, se van a distinguir en ello, sobre todo después del Concilio Vaticano II, 1965, que rompe con las normas de la Deus Scientiarum Dominus y de la Aeterni Patris.

Este progreso y renovación de la lógica se hace notar con la aparición de numerosos manuales tanto en España como en Iberoamérica. A los conocidos de la década anterior, sobre todo, A. Tarski, Introducción a la lógica y' a la metodología de las ciencias, ${ }^{24} \mathrm{~J}$. Ferrater Mora y H. Leblanc, Lógica matemática, ${ }^{25} \mathrm{~W}$. van Orman Quine, El sentido de la nueva lógica ${ }^{26}$ hay que añadir ahora, entre otros, los de W. Ackermann y D. Hilbert, Elementos de lógica, 1962, ${ }^{27}$ M.R. Cohen y E. Nagel, Introducción a la lógica y al método científico, $1968,{ }^{28} \mathrm{H}$. Curry y R. Feys, Lógica combinatoria, $1967,{ }^{29} \mathrm{~J}$. Dopp, Nociones de lógica formal, $1969,{ }^{30} \mathrm{~J}$. Ferrater Mora y H. Leblanc, Lógica matemática, ${ }^{31} \mathrm{~J}$. Ladrière, Limitaciones internas de los formalismos, 1969, ${ }^{32}$ M. Sánchez Mazas, Fundamentos matemáticos de la lógica formal, $1963,{ }^{33}$ P.F. Strawson, Introducción a una teoría de la lógica, $1969,{ }^{34}$ P. Suppes, Introducción a la lógica simbólica, $1966 .{ }^{35}$ Es en esta década cuando se traduce la Historia de la lógica formal de I.M. Bochenski, $1968^{36}$ y su Introducción al pensamiento filosófico. ${ }^{37}$

Dentro de aquel esfuerzo de renovación es donde adquiere y cobra sentido la obra de esta década de Vicente Muñoz. Ésta se articulará, ahora, en torno a tres núcleos o ejes principales. El primero, en torno a la lógica moderna y la lógica escolástica. El segundo, caracterizado por las visiones panorámicas que ofrecerá de la lógica. Y el tercero, en torno al filósofo Ángel Amor Ruibal, 1869-1930.

Actas II, p. 247.

19 Ibidem, p. 245.

20 Ibidem, p. 235.

21 Ibídem, p. 233.

22 Ibídem, p. 224.

23 Barcelona, Herder, 1967.

24 Trad. de T. Rodríguez Bachiller y J.R. Fuentes, Buenos Aires, Espasa Calpe, 1951, reimpresiones, ahora, 1968,

25 México, FCE, 1955, 1962, 1965, 1957, 1968...

26 Trad. de Mario Bunge, Buenos Aires, Nueva Visión, 1958.

27 Trad, de Víctor Sánchez de Zavala, Madrid, Tecnos, 1962, 1968, 1975.

28 Trad. de Néstor Míguez, Buenos Aires, Amorrortu, 1968, 1971, 1973...

29 Trad. de Manuel Sacristán, Madrid, Tecnos, 1967.

30 Trad. de N. Peña y P. de la Cruz, Madrid, Tecnos, 1969.

31 México, FCE, 1955, reimpresiones, ahora, 1962, 1965, 1967, $1968 .$.

32 Trad. de J.L. Blasco, Madrid, Tecnos.

33 Caracas. Imprenta Universitaria.

34 Trad. de J. Ameller V., Buenos Aires, Ed. Nova.

35 Trad. de Gabriel Aguirre Carrasco, México, Compañía Editorial Continental; 1966, 1969, 1970, 1971, 1973.

36 Trad. de Millán Bravo Lozano, Barcelona, Herder, 1968, 1976. Edcición alemana, 1956.

37 Trad. de Daniel Ruiz Bueno, Barcelona, Herder, 1963, 1965, 1970. 
Estos núcleos expresan lo que ha sido su formación en Lovaina y Norteamérica. Por una parte, el descubrimiento que ha hecho de la lógica moderna y la nueva mirada que proyecta sobre la lógica escolástica. Y, por otra, la revalorización que con ocasión del centenario del nacimiento de Amor Ruibal hará de su filosofía, Introducción filosófica al primer centenario del nacimiento de Angel Amor Ruibal (1869-1969), ${ }^{38}$ y que será objeto de posteriores estudios.

La lógica escolástica y la lógica moderna, tal como se estaban enseñando tanto en Europa como en Estados Unidos y Canadá, ya no son dos lógicas enfrentadas. Por ello, Muñoz Delgado va a intentar, según ese nuevo modo de considerar la lógica, establecer puntos de encuentro entre ambas lógicas, dos lógicas distintas pero ambas necesitadas una de otra. Este planteamiento metodológico será uno de los que irá alentando toda su investigación posterior en el campo de la lógica y de la historia de la lógica.

Donde mejor queda realizada esta nueva metodología y perspectiva es en el libro que ahora publica: Lógica matemática y lógica filosófica, 1962. ${ }^{39}$ Fue uno de los más estimados por el propio Muñoz, y en años posteriores quería hacer una reedición de él. Servía para poner las bases metodológicas a fin de procurar un acercamiento a la lógica moderna (matemática) y a la escolástica. En él se analiza la naturaleza de ambas lógicas y se señalan las líneas desde las cuales debería acometerse su investigación, esto es, estamos ante dos familias de lógicas atravesadas por dos modos de saber y operar.

A mi modo de ver, este importantísimo trabajo contiene las claves para entender globalmente la obra de Vicente Muñoz y las tareas urgentes que había que acometer, o, mejor, las tareas pendientes que cada lógica debería cumplir y a las que él se iba a consagrar. Desde esta perspectiva global de la lógica, que se ha hecho y se hace historia en la propia historia, ya sólo habrá lógica que se expresa a través de una pluralidad de lógicas y de una diversidad de lógicos de diferentes credos y naciones en la riqueza de cada cirscunstancia histórica.

Dirá, así, en el «Prólogo y presentación» de esta obra:

El intento de este trabajo es mostrar la verdadera naturaleza de la lógica matemática y de la lógica aristotélico-escolástica.

La logística es esencialmente una matemática, por su objeto y por sus procedimientos; la lógica escolástica es una lógica filosófica, por su objeto, por su método y por su ideal cognoscitivo.

Delimitados así los campos, es fácil valorar las críticas que mutuamente se han hecho esas dos familias de lógicas, en general desprovistas de valor, por proyectar el modo de conocer matemático en el modo conceptual, y viceversa.

Para hacer ver mejor nuestra tesis, establecemos después una comparación en puntos fundamentales, para corroborar que son dos saberes distintos, que no se contradicen y que se pueden ayudar mutuamente. ${ }^{40}$

Y cerrará el libro con la siguiente «Conclusión final»:

Una vez que hemos estudiado la naturaleza de la lógica matemática y de la lógica escolástica, las objeciones que mutuamente se hacen, indicando de modo general que las divergencias proce-

38 Estudios, 24 (1968), pp. 367-401. Para este aspecto remito al importante trabajo del prof. Cesar Raña Dafonte, que aparece en esta misma Revista Española de Filosofía Medieval.

39 Madrid, De. Revista Estudios, 1962, 288 pp. Nada más de venir de Lovaina había publicado, «Lógica escolástica y lógica moderna», Estudios, 12 (1956), pp. 115-124. «La lógica antigua y medieval a la luz de la logística», Salmanticensis, 4 (1957), pp. 503-541. Era la nueva consideración de la lógica escolástica que se hacía en Lovaina.

40 Lógica matemática, p. 9. 
den de la diferente perspectiva en que una y otra se colocan, hemos hecho una comparación más detenida entre ambas en esta tercera parte. La conclusión es siempre la misma.

La lógica matemática es esencialmente una matemática; la lógica filosófica es dependiente de una filosofía a la que está íntimamente ligada. En nuetro caso, la lógica escolática está dependiendo de las nociones más importantes de la filosofía escolástica.

De ahí que entre ambas se puedan encontrar paralelismos y extremos de comparación, pero no es posible la reducción de una a otra. ${ }^{41}$

Es en esta doble tarea en la que se comprometerá Vicente Muñoz Delgado, desde ésta que parece esconder una proclama programática: recuperar la rica tradición lógica escolástica, y cultivar la lógica moderna y la ciencia en el límite de sus consecuencias, sabiéndose situar en la frontera del conocer humano para procurar un más compendioso pensar y filosofar.

Señalo ahora algunos de los trabajos más relevantes relativos a cada uno de los ejes o núcleos indicados.

\subsection{Lógica escolástica e historia de la lógica}

De entre todos ellos destacaría el conocido y citado libro La lógica nominalista en la Universidad de Salamanca (1510-1530), $1964 .^{42}$ En esta obra aparece ese nuevo modo de tratar y de presentar la lógica escolástica, lo cual conlleva su revalorización y descubrimiento.

Pero, para el caso concreto de la Universidad de Salamanca, esta investigación va a significar una inflexión complementaria con respecto a los estudios e imagen que, entre otros, V. Beltrán de Heredia proyectaba o se había ido forjando con rèspecto a dicha Universidad y su esplendor teológico. La Universidad del Tormes en estos contextos del siglo XV y XVI no sólo había cultivado la teología sino, también, y de modo destacadísimo la lógica y la ciencia.

De otra parte, esta obra retomaba el pulso de un ya viejo artículo suyo de 1954, «La enseñanza de la lógica en Salamanca durante el siglo XVI», ${ }^{43}$ y se situaba en sintonía con G. Beaujouan y su La science anglaise dans les bibliothèques de Salamanque au XVème siècle ${ }^{44}$ así como con la importante Manuscrits scientifiques médiévaux de l'Université de Salamanque et de ses «Colegios Mayores». ${ }^{45}$ Esta dirección será una de las líneas que marcará, también, la obra de Muñoz Delgado y que se tornará en llave maestra para toda una serie de investigaciones que se irán abriendo y completando en el tiempo. Entre ellas, las de Cirilo Flórez Miguel, José Luis Fuertes Herreros, A. Carabias Torres, L.E. Rodríguez-San Pedro, Angel D'Ors... Con ello, la Universidad de Salamanca quedaba engarzada en la onda renovadora que durante el Renacimiento se extiende por Europa, y de modo concreto en y a través de las grandes universidades de la cristiandad: París, Salamanca, Oxford, Cambridge, Padua, Bolonia y Alcalá. 


\subsection{Lógica moderna}

Sus aportaciones de esta década van a girar en torno a los distintos ámbitos de la lógica, en orden a la filosofía y lenguaje, matemática, y en orden a la inducción y método.

De entre las obras, merecen destacarse:

1961 De la axiomática a los sistemas formales.

1962 Lógica matemática y lógica filosófica.

1963 La cantidad en la lógica tradicional y en la lógica matemática.

1965 Los lenguajes formalizados de la lógica moderna.

Aunque la primera de éstas bien merecería detenernos en ella, sin embargo, considero que es mejor completar un aspecto que aparece en la segunda, esto es, en la Lógica matemática y lógica filosófica. Me refiero a la bibliografía que acompaña a esta obra, y que va de las páginas 273 a la 288 con unos 255 títulos aproximadamente.

Esta bibliografía supone el mejor estado de la cuestión que uno puede imaginar y utilizar para reconstruir el estado de la lógica a comienzos de los años 60 . Vicente Muñoz sabe recoger lo último y más selecto de la lógica en estos momentos. Hay en ella una muy eficiente cartografía de las tendencias en lógica. Ahí están todos los que tienen que estar: Ackermann, Bell, Bernays, Beth, Bochenski, Boole, Bourbaki, Carnap, Cohen, Curry, Church, Dopp, Feys, Frege, García Bacca, Nagel, Russell, Schroeder, Suppes, Tarski, Whitehead..., entre otros.

\subsection{Visiones panorámicas sobre el estado de la lógica}

Tres visiones va a ofrecer sobre el estado de la lógica en Norteamérica, Alemania y Europa, en otros tantos trabajos, tras su vuelta a Salamanca:

1961 El problema de la lógica en los medios neoescolásticos norteamericanos de la segunda postguerra.

1962 El cultivo de la lógica matemática en la Alemania de la postguerra.

1967 El neopositivismo en su fase europea.

Esta línea, ya en este mismo año 67, será continuada sobre todo con abundantes trabajos referidos a España, Portugal e Iberoamérica a partir de los años 70, como luego veremos.

\section{LÓGICA, CIENCIA Y FILOSOFÍA EN PERMANENTE RENOVACIÓN Y PERFECCIONAMIENTO, 1970-95}

Con la década de los años 70 se van a desarrollar y perfeccionar las líneas anteriores. A las 87 publicaciones anteriores y unas 120 recensiones hay que sumar los 153 trabajos nuevos, sin contar las aproximadamente 670 recensiones que desde el año 70 al 95 irán surgiendo.

Quizá lo más destacable de estos años en la génesis de su obra y pensamiento sea el comprobar cómo la renovación emprendida en la década anterior en torno a la lógica escolástica e historia de la lógica, lógica moderna y visiones panorámicas, va a ir siendo perfeccionada desde una perspectiva mucho más amplia, desde la ciencia y la filosofía. Y cómo, ahora, podemos aglutinar el conjunto de su obra en torno a cinco ejes principales, que engloban a los anteriores y que, a la vez, sirven para vertebrar y aglutinar de un modo nuevo el conjunto de los trabajos de estos 25 últimos años. Los ejes, según un orden cronológico, son:

a) Los Congresos Internacionales de Historia de las Ciencias Eclesiásticas en España.

b) Los Congresos Internacionales de Lógica, Metodología y Filosofía de la Ciencia. 
c) Los Congresos Internacionales de Filosofía Medieval.

d) Seminario de Historia de la Filosofía Española e Iberoamericana.

e) Los Congresos Nacionales de Filosofía Medieval.

Hay otros dos ejes que se nos filtran continuamente en los anteriores y que, inclusive, parecen servir de amalgama en la obra de Vicente Muñoz. Me refiero a la Universidad de Salamanca y a la Orden de la Merced, que ya estaban presentes desde sus primeros escritos. A ambos les dedicaré su apartado correspondiente:

f) Vicente Muñoz y la Universidad de Salamanca.

g) Vicente Muñoz y la Orden de la Merced.

Y cerraré todo este apartado acercándome al manual de lógica que Muñoz Delgado publicó para uso de clase. A través de él aparecerá, no ya la figura del investigador, sino la del profesor y la renovación que día a día se pretendía y que iba fraguando no sólo en el contexto salmantino sino en el de la Universidad española. Será:

h) Lecciones de Lógica (I) y (II).

Cada uno de dichos ejes parece marcar, en gran medida, las preocupaciones e investigaciones de estos años. A partir de los 70 se va acentuando su dedicación al cultivo y descubrimiento del patrimonio de la lógica escolástica, sobre todo la desarrollada en España. Es como si a través de los congresos internacionales se le hubiera mostrado, por una parte, la urgencia que había de rescatar dicha herencia lógica, y, por otra, que dichos congresos eran el marco y foro adecuado tanto para dar a conocer la lógica cultivada en España, como para comprender las aportaciones de ésta al acervo común de la lógica y de la cienca en los distintos momentos históricos.

\section{CONGRESOS INTERNACIONALES DE LA HISTORIA DE LAS CIENCIAS EN ESPAÑA}

Estos congresos, celebrados en Salamanca en 1965, 1970 y 1975 bajo los auspicios del Instituto de Historia de la Teología Española de la Universidad Pontificia de Salamanca, del cual Vicente Muñoz era director de la Sección de Filosofía, tenían como finalidad «ofrecer material de fuentes y bibliografía para la historia cultural de España». Las actas de dichos tres congresos aparecieron bajo el título Repertorio de Historia de las Ciencias Eclesiásticas en España en 7 volúmenes.

Las aportaciones de Muñoz Delgado a ellos fueron las siguientes:

I, 1965 «Fuentes impresas de la lógica hispano-portuguesa en el siglo XVI», y «La Teología entre los mercedarios españoles hasta 1600». Dichos trabajos aparecieron, repectivamente, en el Repertorio, I, 1967, y III, 1971.

II, 1970 «La lógica hispano portuguesa hasta 1600. Notas bibliográfico doctrinales». ${ }^{46}$

Este importante trabajo ofrece una visión panorámica «de la historia de la lógica desde el período romano hasta el siglo XVI. La lógica se toma con la amplitud suficiente para dar una visión 
retrospectiva de los problemas que interesan al lógico actual en las variadas dimensiones que contribuyen al desarrollo de esta disciplina, como son la relación entre lógica y retórica, lógica y gramática, lógica y ciencia, señalando cuidadosamente la aportación española y portuguesa». ${ }^{47}$

III, 1975 «Ciencia y filosofía de la naturaleza en la Península Ibérica (1450-1600)».48

En este, igualmente, importante trabajo se enumeran «los comentarios a la física y astronomía antiguas, en contraste con las nuevas aportaciones físicas y matemáticas, descubrimientos, etc., que van preparando la aparición de la ciencia y el método científico moderno. Constituye un complemento a los trabajos recientes de historia de la ciencia hispano-potuguesa, donde se descuida la aportación de las cátedras de filosofía de la naraturaleza y astrología, en las que se advierte la presencia de elementos antiguos que van siendo superados por el progreso y la crítica». ${ }^{49}$

Estas investigaciones, junto con otras anteriores reseñadas, servirán para la nueva mirada que se ha ido forjando en torno al Renacimiento lógico, filosófico y científico que se produce en España, y en concreto en las Universidades de Salamanca y Alcalá.

\section{LOS CONGRESOS INTERNACIONALES DE LÓGICA, METODOLOGÍA Y FILOSOFÍA DE LA CIENCIA}

Por los trabajos presentados a ellos percibimos la perspectiva y metodología que en torno a la lógica, ciencia y filosofía late en muchos de los estudios dedicados a temas y autores de los siglos XV y XVI. A través de todos ellos es posible reconstruir la lógica, su historia y sus problemas.

III, 1967, Amsterdam. Asiste.

IV, 1971, Bucarest «The formal Logic in Spain (1450-1550)».

V, 1975, Londres-Ontario «The "consequentia" in the works of spanish logicians (14701550)».

VI, 1979, Hannover «Logic, humanism and science in Salamanca (1490-1554)».

VII,1983, Salzburgo «La "Consequentia" y sus divisiones generales en Juan de Oria».

\section{LOS CONGRESOS INTERNACIONALES DE FILOSOFÍA MEDIEVAL}

En las aportaciones a estos Congresos late la misma perspectiva que en los anteriores y en torno a ellos se aglutinan los numerosos trabajos que va publicando.

V, 1973, Madrid-Córdoba «El "Compendio de lógica" (1351) de Nicolás Eymerich».

VI, 1977, Bonn «Meaning in some Spanish Logicians (1500-1550)».

VII,1982, Lovaina «Lógica, conocimiento y hombre en Juan de Oria (1518)».

VIII,1986, Helsinki «Los "Insolubilia" en la Epistemología de Juan de Oria».

47 «Prólogo», Repertorio, IV, 7.

48 Repertorio, VII, Salamanca, 1979, pp. 67-148.

49 «Prólogo», Repertorio, VII, 8. 
Esta dedicación a Juan de Oria, que aparece tanto en los Congresos de Lógica como en los de Filosofía Medieval, culminarán con los dos libros que sobre él escribirá: Johannis de Oria Opera Logica (1518). Summularum volumen primum: Tractatus de conceptu et modo concipiendi. Tractatus Dialecticae. Tractatus propietatum circa elementa dialecticae, $1987 .{ }^{50}$ Johannis de Oria Opera Logica: Tractatus de Enuntiatione, $1990 .{ }^{51} \mathrm{Y}$ tendrá su continuidad en la Revista de Filosofía Medieval, 1994: «El "Tractatus consequentiarum" (1518) en la lógica de Juan de Oria», 49, 1 (1994), pp. 49-60.

\section{SEMINARIO DE HISTORIA DE LA FILOSOFÍA ESPAÑOLA E IBEROAMERICANA}

Iniciado a partir de 1978 y coordinado por Antonio Heredia Soriano se celebra regularmente cada dos años en el marco de la Facultad de Filosofía de la Universidad de Salamanca. En septiembre de 1996 se celebraba su décima edición.

Vicente Muñoz ha particiapado en él desde su creación, presentando los siguientes trabajos, todos ellos recogidos en las actas:

II, 1980 «Para la historia de la lógica en España e Iberoamérica».

III, 1982 «Ortega y Gasset y el proyecto de una lógica de la razón vital».

IV, 1984 «Los mercedarios en el Perú durànte el período español».

V, 1986 «Profesores mercedarios de la Real y Pontificia Universidad de México hasta 1821».

VI, 1988 «Los estudios de Filosofía en la Provincia mercedaria de México».

VII, 1990 «La obra americana de Augusto Pescador».

VIII, 1992 «Nuevas direcciones en la lógica Iberoamericana».

Repárese cómo a través de este Seminario se nos filtra, ahora, ese otro eje que se va haciendo presente y que va aflorando: La Orden de la Merced y su contribución a la filosofía, que supone una nueva mirada y reflexión sobre la misma. De otro lado, continúa en su dedicación a la lógica en España e Iberoamérica.

\section{LOS CONGRESOS NACIONALES DE FILOSOFÍA MEDIEVAL}

Impulsados por la Sociedad de Filosofía Medieval (SOFIME), con sede en el Departamento de Filosofía de la Universidad de Zaragoza, siendo su presidente y secretario, respectivamente, Joaquín Lomba Fuentes y Jorge M. Ayala Martínez, se inician a partir de diciembre de 1990. Se celebran cada cuatro años y se publican las actas de cada Congreso. En diciembre de de 1994 se celebra el II Congreso. Por su parte, la Sociedad de Filosofía Medieval publica la Revista de Filosofía Medieval bajo la dirección de Jorge M. Ayala.

Muñoz participará en el I Congreso, 1990, «La Lógica medieval». Pero al II, 1994, ya no asistirá, pues había sido operado de cataratas y no se atreve a abandonar Salamanca. Sin embargo, en

50 Madrid, CSIC, 1987, 326 pp. Preparación del texto, introducción, notas e índices por Vicente Muñoz Delgado.

51 Madrid, CSIC, 1990, 383 pp. Edición del texto, introducción, notas, índices y texto por Vicente Muñoz Delgado. 
dicho año envía a la Revista de Filosofía Medieval su artículo «El "Tractatus consequentiarum" (1518) en la lógica de Juan de Oria», que ya señalé anteriormente.

En torno a estos ejes se aglutinan los 153 trabajos de esta etapa, relacionados con la lógica y su historia, la ciencia y la filosofía. Y es ahí, y desde esta perspectiva, desde la cual adquieren significado los distintos estudios que van por autores tan diversos como algunos poco conocidos, entre los cuales señalo: José Blasco, Alonso de Córdoba, Bernando Jordán, Pedro de Campis, Juan Hidalgo, Pablo de Venecia, Jerónimo Pérez, Pedro de Castrovol, Francisco de Cristo, Luis de Lemos, Ángel Estanyol, Cipriano Benet, Juan Aznar, Celestino del Santísimo Sacramento, Alonso de la Vera Cruz, Rodrigo Sánchez de Arévalo, Pedro Núñez, Juan Martínez Nieto, Pedro de Osma, Pedro de Espinosa, Juan de Oria, Luis de Aparicio, Martín de Samunde, Agustín de Esbarroya, Juan García Rodríguez, Ignacio de Andrade y Ribóo, Jacinto de Angueira, Fernando de Enzinas, Francisco Salvador Gilabert, Pedro de Celis, Hernando de León, Francisco Zumel, Juan Gómez...

Igual acontece con la visiones panorámicas que sobre la lógica y su historia, la ciencia y la filosofía, o sobre algún problema, o sobre una corriente o grupo de autores nos va a ofrecer. Además de las ya señaladas, en estos mismos años 70-95 tenemos:

1970 La lógica de los españoles en París (1500-1525).

1973 La lógica formal en España (1350-1540).

España en la historia de la lógica prerrenacentista (1350-1550).

1974 La lógica formal y su dimensión histórica.

1975 Introducción al patrimonio escolástico de la lógica.

1976 Peripatetismo y filosofía moderna en España.

1979 Consideraciones sobre la lógica y' su historia.

1980 Lógica, ciencia y humanismo en la renovación teológica de Vitoria y Cano.

1981 Lógica, ciencia y humanismo en Salamanca (1480-1550).

1982 Lógica hispano-portuguesa e iberoamericana en el siglo XVII (bibliografia, fuentes impresas y manuscritas).

1986 Nacionalismo, lógica y humanismo.

1988 La crítica de de los humanistas a la ciencia y a la lógica de la escolástica tardía.

1990 Nominalismo, lógica y humanismo del siglo XIV al XVI.

1991 Filosofía, teología y humanidades en la Universidad de Salamanca durante los estudios de San Juan de la Cruz (1564-1568).

La lógica moderna en los primeros escritos de Juan David García Bacca.

1992 El pensamiento lógico en Iberoamérica.

En esta misma línea, también, hay que destacar las aportaciones de Muñoz Delgado al Lexikon für Theologie und Kirche, 1965, artículo, «Zumel»; al Historisches Wörterbbuch der Philosophie de J. Ritter, vol. I, 1971, términos: «Ampliatio», «appellatio», «ascensus et descensus», «autofalsifikation», «connotatio»; al vol. II, 1972, «demonstrabel», «denominatio»; vol. IV, 1976, «Konsequenz»; y al Diccionario Teológico. El Dios cristiano, 1992, artículo, «Trinidad y Lógica».

\section{VICENTE MUÑOZ Y LA UNIVERSIDAD DE SALAMANCA}

Como he indicado anteriormente, voy a reparar en este aspecto y dedicarle un apartado específico, dejando agrupados los trabajos, aunque no todos, que han girado en torno a la Universidad 
de Salamanca, a pesar de que algunos ya los haya señalado. He de decir también que en los estudios que Vicente Muñoz hace de la Universidad Salmantina se nos filtra muchas veces la de Alcalá, ya que ésta aparecía como el alter ego de la de Salamanca en esos contextos de emulación y oposición que se vivieron en el Renacimiento.

Es casi impensable la obra de Muñoz Delgado sin esta vinculación con la Universidad del Tormes, por los trabajos vinculados directa o indirectamente con ella, y, a la inversa, la investigación sobre la Universidad de Salamanca quedaría sesgada si se olvidaran los estudios y las nuevas perspectivas que Muñoz le supo abrir e imprimir.

Además de aquellas obras primeras, El influjo del entendimiento sobre la voluntad según Francisco Zumel (†1607), 1950, Zumel y el molinismo, 1953, que supusieron un acercamiento a la Universidad de Salamanca y a la polémica de auxiliis que se vivía en ella, hay que destacar, ya desde una nueva perspectiva:

1954 La enseñanza de la lógica en Salamanca durante el siglo XVI.

1960 Domingo de Soto y la ordenación de la enseñanza de la lógica.

1963 Narciso Gregori y la lógica del humanismo en salamanca durante la segunda mitad del siglo XVI.

La exposición sumulista de la doctrina silogística de Domingo de San Juan de Pie del Puerto (†1540).

1964 Lógica formal y filosofía en Domingo de Soto.

La lógica nominalista en la Universidad de Salamanca (1510-1530).

1965 Domingo Báñez y las Súmulas en Salamanca a fines del siglo XVI.

Domingo de San Juan de Pie del Puerto (†1540) y su obra acerca de las "Oppositiones» entre proposiciones.

1967 La lógica en Salamanca durante la primera mitad del siglo XVI.

1972 Los «principia Dialectices» (1519) de Alonso de Córdoba.

1975 El Maestro FR. Juan Martínez Nieto. El Colegio de la Veracruz de Salamanca y la Universidad en la segunda mitad del XVIII.

Juan de Aguilera (1560-61) y su «Ars memorativa» (1536).

1976 Peripatetismo y filosofía moderna en España.

Juan Martínez Nieto (†1806) y la Universidad de Salamanca.

$E l$ «Curso de filosofia» de la Universidad de Salamanca (1772-84).

1977 Un colegio de Artes para la Universidad de Salamanca en dos «Dictámenes» (1789, 1796) de Fr. Juan Martinez Nieto.

Filosofía y' Teología en Salamanca (1772-1796) a través del pensamiento de Juan Martínez Nieto.

1978 Un colegio de filósofos y matemáticos para la Universidad de Salamanca en dos «Dictámenes» $(1789,1796)$ de Juan Martínez Nieto.

1980 Lógica, ciencia y humanismo en la renovación teológica de Vitoria y Cano.

1981 La Universidad de Salamanca (1778) y los «Elementa» de Juan Díaz de Gamarra. Lógica, ciencia y humanismo en Salamanca (1480-1555).

1986 Nominalismo, lógica y humanismo.

1990 Nominalismo, lógica y humanismo del XIV al XVI.

1991 Filosofia, teología y humanidades en la Universidad de Salamanca durante los estudios de San Juan de la Cruz (1564-1568). 
En este contexto salmantino hay que resaltar las obras sobre Juan de Oria, así como los trabajos aparecidos en el Repertorio sobre lógica y filosofía de naturaleza, ya que inciden en gran medida en la Universidad de Salamanca y en la de Alcalá.

Sobre la Universidad de Alcalá, tenemos específicamente, La lógica en la Universidad de Alcalá durante la primera mitad del siglo XVI, 1968.

\section{VICENTE MUÑOZ Y LA ORDEN DE LA MERCED}

Tanto por la importancia de las aportaciones de la Orden de la Merced a la lógica, ciencia, filosofía y teología, como por la propia consagración y fidelidad a dicha orden, Vicente Muñoz le va a dedicar una atención especial en la etapa que nos ocupa, de 1970 a 1995.

No me refiero ahora a estudios sobre autores concretos de la propia orden, como podía ser el caso de Zumel, o Domingo de San Juan de Pie del Puerto, sino a trabajos sobre la orden en general. Así, además de otros ya apuntados en el Seminario de Historia de la Filosofía Española e Iberoamericana, destaco:

1971 La Teología entre los mercedarios españoles hasta 1600.

1973 La filosofía entre los mercedarios del siglo XVII y XVIII según unos inéditos de Palma.

1978 Los mercedarios de la Veracruz en el "Diario de un estudiante» (1603-1607) de Salamanca.

La producción filosófica de los mercedarios en el siglo XVIII.

1982 Los mercedarios y la filosofía en el siglo XVIII a través de una sintesis de 1978.

1984 La Veracruz de Salamanca y dos primeros profesores de la Universidad.

1988 Profesores mercedarios de la Real y Pontificia Universidad de México hasta 1821.

\section{LECCIONES DE LÓGICA (I) Y (II)}

Cerraré este apartado refiriéndome a su manual de lógica, Lecciones de Lógica (I), Salamanca 1972, y Lecciones de Lógica (II), Salamanca 1974, por haber sido publicado en esta última etapa y por haber sido libro de texto, y de obligado estudio, de muchos universitarios.

Hay que enmarcar dicho manual en ese compromiso por impulsar y contribuir a la necesaria renovación de la lógica que Vicente Muñoz se había propuesto. Uno de los mejores medios eran las aulas universitarias. «Estas Lecciones de Lógica en dos volúmenes -dirá Muñoz Delgado- responden a los cursos que hace muchos años vengo explicando en la Universidad Pontificia en permanente renovación y perfeccionamiento [...] Con mi mejor afecto pienso en los numerosos alumnos que, a lo largo de tantos años, me han acompañado en la tarea cotidiana de renovar un saber dinámico y progresivo, con tantas aplicaciones al mundo de la ciencia y de la técnic» ${ }^{52}$

Estas Lecciones compendiaban toda su tarea anterior, así como el modo de entender y de aproximarse a la lógica: un intento de acercar, desde la diferencia, la lógica escolástica y la lógica mate-

52 Lecciones I, pp. 3-4. Para una panorámica del estado de la lógica en Epaña, véase, Rodolfo Fernández González, «Mapa actual de la lógica en España», en Actas II, pp. 329-348. 
mática. Como él mismo señala: «Lecciones de Lógica, Lógica matemática y lógica filosófica, Lógica hispano-portuguesa hasta 1600 (Salamanca 1972), nuestro Programa de Lógica y' Metodología, así como todos mis trabajos, suponen una actitud consciente y muy abierta a toda la problemática filosófica e histórica». ${ }^{53}$

De modo general, hay que situar también esta obra en la línea de renovación emprendida en España en la década de los años 60. Los manuales en la nueva de los 70 se van a ir sucediendo, intentando llegar a un público universitario cada vez más numeroso, crecido en parte, asimismo, por las nuevas Secciones de Filosofía que surgen. Y así, entre otros, tenemos:

1970 Benson Mates, Lógica matemática elemental, trad. de Carmen G. García Trevijano, Madrid.

1971 Jesús Mosterín, Teoría axiomática de conjuntos, Barcelona.

1972 Alfredo Deaño, Lógica simbólica y lógica del lenguaje ordinario, Madrid.

1973 M. Sánchez Mazas, Cálculo de normas, Madrid.

G.E. Hughes y M.J. Creswell, Introducción a la lógica modal, Madrid.

1974 Manuel Garrido, Lógica simbólica, Madrid.

Alfredo Deaño, Introducción a la lógica. I. Lógica de enunciados, Madrid.

David Mitcheli, Introducción a la lógica, Barcelona.

1975 Alfredo Deaño, Introducción a la lógica. II. Lógica de enunciados, Madrid.

Pascual Martínez Freire, Lógica matemática. Primeras lecciones, Madrid.

A. González Carlomán, Lógica axionática, Oviedo.

1976 María Luisa della Chiara Scabia, Lógica, Barcelona.

L. Couturat, El álgebra de la lógica, Madrid.

1977 H. Seiffer, Introducción a la lógica, Barcelona.

1978 P.H. Nidditch, El desarrollo de la lógica, trad. de Carmen G. Trevijano, Madrid.

A éstos hay que añadir las reediciones que se hacen de manuales de la década anterior, entre ellos, el Ackermann-Hilbert, Agazzi, Cohen-Nagel, Ferrater Mora-Leblanc, Menne, Quine, Sacristán, Suppes o Tarski.

Posiblemente las Lecciones de Lógica fueran algo más que un manual. Tendían un puente entre la lógica escolástica y la lógica matemática: «La lógica greco-escolástica consta de dos grandes aportaciones: la aristótelica [...] y la megárico-estoica»; 54 a partir de ahí, sentada esa base, que compendía todo un modo de entender la lógica, se descubrían los distintos problemas y relaciones, y se adentraban en la lógica.

Y a través de la lógica se Muñoz Delgado se acercaba a la última filosofía, a la dialéctica, al marxismo, a la filosofía del lenguaje, al estructuralismo, al positivismo, a las matemáticas, a la ciencia, al derecho, a la ética, a las ciencias sociales, a la informática..., a la historia de la lógica y, además, se hacía una nueva reinterpretación de ésta, porque «el hombre actual tiene una especial conciencia del dinámico devenir histórico del saber que es continuamente perfectible. El formalismo lógico se ha interpretado filosóficamente de una manera estática. Pero la lógica como un saber vivo e histórico deberá tener muy presente su evolución en el pasado y su posible transformación futura». 55 


\section{CONCLUSIÓN}

He intentado ofrecer una aproximación a la obra de Vicente Muñoz Delgado, destacando las diversas etapas que jalonan su obra y pensamiento, así como los núcleos y las claves sobre las que se vertebra. Los finales de los años 40, como punto de partida o de toma de conciencia de una situación de la lógica de la que había que salir. La del 54 al 60, con sus nuevas perspectivas. La de los años 60 al 70, con ese compromiso por impulsar la necesaria renovación; y la del 70 al 95, en la que está en permanente renovación y perfeccionamiento. En cada una de estas etapas hemos visto cómo su obra se ha ido estructurando y aglutinando en torno a diversos núcleos, que sirven para contener su dedicación a la lógica, a la ciencia y a la filosofía.

Ha sido un camino de renovación, que, solidariamente, con muchos otros ha acabado transformando el hacer lógico, filosófico y científico de la España de la postguerra, para llegar al florecimiento que se fue fraguando y que comenzó a vivirse a partir de los años 70 .

José Luis Fuertes Herreros

Departamento de Filosofía

Campus Unamuno. Edif. FES

Universidad de Salamanca

E-37.007 SALAMANCA 